\title{
The geriatric canon
}

\author{
Renzo Rozzini ${ }^{1,2}$ \\ ${ }^{1}$ Poliambulanza Foundation, Istituto Ospedaliero, Brescia; ${ }^{2}$ Società Italiana Cardiologia Geriatrica, Florence, Italy
}

\begin{abstract}
Over the years Geriatrics and Gerontology have developed a language shared by most clinicians and researchers who unequivocally defines the health conditions of aging people: one could speak of "geriatric canon", i.e. the set of fundamentals (paradigms, principles, standard reference vocabulary) of geriatric knowledge, the heritage from which they draw inspiration and from which the actions for the care of the elderly can be driven, the scientific works for the study of their health. The aim of this paper is to describe and report the most important terms of the geriatric canon, in a simplified way, in order to establish a more precise use of geriatric terminology that can be easily utilized by the cardiologists, or other specialists who takes care of elderly patients, without depriving them of their clinical significance, and becoming heritage of ordinary medical language.
\end{abstract}

\section{Introduction}

Consider a 70-year-old woman with a surgical indication of knee arthroprosthesis, suffering from hypertensive heart disease, diabetes mellitus type 2, chronic renal failure and anxiety disorder, poorly able to understand Italian language and caring for her 75year-old husband disabled for a previous stroke.

The cardiologist will pay attention to her heart disease, considering diabetes, renal failure and anxiety disorder as comorbidities. Her general practitioner could describe the patient "suffering from multimorbidity", paying equal attention to hypertensive heart disease, diabetes, and anxiety. The burden of multiple coex-

\footnotetext{
Correspondence: Renzo Rozzini, Fondazione Poliambulanza, Istituto Ospedaliero, Via Bissolati 57, 25124 Brescia, Italy.

E-mail: renzo.rozzini@poliambulanza.it
}

Key words: Comorbidity; multimorbidity; frailty; disability; complexity.

Received for publication: 3 January 2019.

Accepted for publication: 12 February 2019.

(C) Copyright R. Rozzini, 2019

Licensee PAGEPress, Italy

Monaldi Archives for Chest Disease 2019; 89:1028

doi: 10.4081/monaldi.2019.1028

This article is distributed under the terms of the Creative Commons Attribution Noncommercial License (by-nc 4.0) which permits any noncommercial use, distribution, and reproduction in any medium, provided the original author(s) and source are credited. isting diseases will be determined by the presence of a number of conditions taking into account their biomedical and functional weight. Finally, the complexity of the patient will in some way be conditioned by her cultural background, by her ability to understand our language, by her living conditions (by her economic availability!) and not least by her role as husband's caregiver.

How many professional figures come into play in the complexity of the care of the patient described, what language they use to communicate with each other, what meaning they attribute to "multimorbidity", "polypathology", "frailty", "disability", "burden of diseases" terms, and yet, how each of them will perceive and understand the intrinsic coherence among the different clinical conditions and will act accordingly? Geriatrics originates within a language, based on complexity, which is currently overlooked. No one has time and patience to bear this complexity: if you need just a few seconds of web consultation to get an answer why should you break your head in some damn analysis or reasoning?

If the complexity, the hallmark of aging, finds little room in the medical mainstream of our day, and if complexity, sometimes perceived as chaos, is poor understood, the clinical language requires an effort to simplify meanings of the words or concepts that are difficult to embrace, without trivializing or deforming reality. Over the years Geriatrics and Gerontology sciences have developed a glossary, shared by most clinicians and researchers in the aging field, who define the health conditions of aged persons: we can consider it the "geriatric canon", i.e. the set of fundamentals (paradigms, principles, standard reference vocabulary) of geriatric knowledge, the heritage from which they draw inspiration and from which must follow the actions for the care of the elderly, the scientific works for the study of his health (Figure 1).

The purpose of this article is to describe and report the most important terms of the geriatric canon, in a simplified way, in order to establish a more precise use of geriatric terminology that can be easily utilized by the cardiologists, or other specialists who takes care of elderly patients, without depriving them of their clinical significance, and becoming heritage of ordinary medical language.

\section{General aspects}

The following unavoidable general aspects of aging need to be considered before approaching an elderly patient:

i) Characteristic sign of aging is the reduced ability to respond to stress.

ii) In elderly person the body's response is what typically generates the symptoms of a disease: it is never so active (this reason explains why a heart disease can be silent).

iii) A disease rarely occurs alone; the involvement of multiple organs and systems is the rule, same as multiple drugs treatment. 
iv) Diseases occur more often as "syndromes" and the definition of the relationship between clinical presentation (syndromic), single pathologies, biological data, response to therapy, cure and care (sometimes due to jatrogenesis, think about the consequences of delirium sedation or physical restraint ...) imposes a unified top-down approach rather than fragmentation into subsystems;

v) The definition of outcomes in elderly patients is not unique and one-way (healing is rarely the main outcome, more often is the prevention or reduction of disability, always the symptoms' relief); it requires a prognosis (more often due patient's health status than single disease severity) that depends on the interaction between physical, mental and social environmental domains [1,2].

Below are the most relevant and frequent terms of the language of geriatrics (the glossary, "The lexicon of geriatrics") to be shared by the medical and surgical disciplines that deal with elderly patients.

\section{Aging}

Systemic consequences of aging are:

1. Changes in body composition. Changes in body composition are the most evident effect of aging: i) the so-called lean body mass, composed predominantly of muscles and visceral organs, decreases; ii) fat mass tends to increase. The lean mass / fat mass ratio changes unfavorably; iii) progressive demineralization and architectural modification occur in bone. Such changes are influenced not only by aging, but also by illness and by lifestyles factors such as physical activities and diet.

2. Balance between energy availability and energy demand. Overall elderly persons have low available energy level and require more energy both at rest and during physical activity. Energy status can be clinically assessed by simply asking the patient about his level of fatigue in carrying out walking or dressing. Older people may consume all their available energy performing the most basic activities of daily living, and consequent fatigue and restriction may lead to a sedentary existence.

3. Modification of the "network" that control homeostasis. The main pathways that control homeostasis involve hormones, inflammatory mediators and antioxidants are profoundly affected by aging (no standard criteria exist that allow the detection and quantification of homeostatic dysregulation as a general phenomenon).

4. Neurodegeneration phenomena. Cortical and subcortical changes are reflected in the high prevalence of "soft" nonspecific neurologic signs, such as slow and instable gait, poor balance, and slow reaction times. Poor performance in a dual task (dual tasks in which a cognitive and a motor task are performed simultaneously) is a marker of reduced overall capacity for central processing, so that simultaneous processing is more constrained [2].

\section{Index condition}

The index condition is the primary disease of interest of the clinical act (it is the specific focus of attention).

An index condition may be somatic (i.e. heart failure) or psychic (i.e. anxiety disorder), may be acute (i.e., myocardial infarction, pneumonia, major depressive disorder) or chronic (i.e., chronic heart failure, type 2 diabetes mellitus, dysthymia). The definition of an index condition is driven by the professional who is managing the disease and his/her point of view.

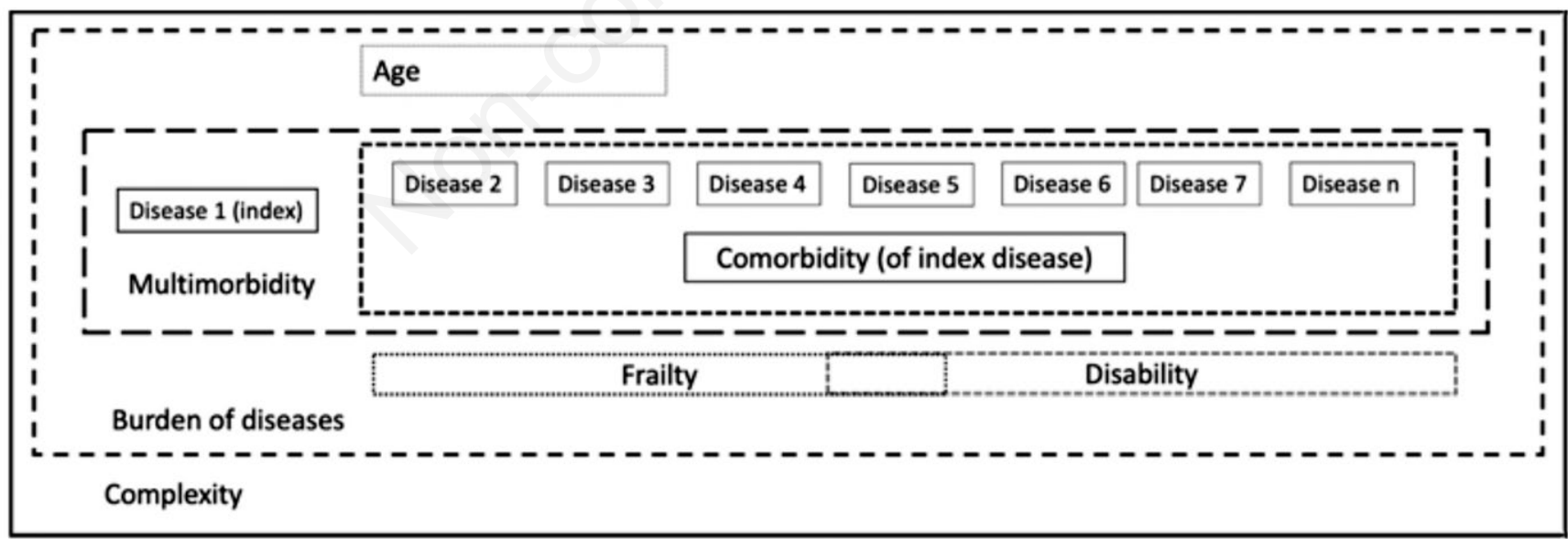

Comorbidity the term refers to pre existing or coexisting distinct additional diseases with respect to an "index" disease

Multimorbidan; The term multimorbidity describes the co-presence of two or more chronic diseases or medical conditions in the same individual without attributing to them a causal role or a priority clinical importance. In a condition of multimorbidity, chronic diseases can be somatic or psychic; concordant; co-occurring and non-concordant; more or less severe. Condition can have a prevalent impact on survival or on disability, a short or a long evolution, a variable impact on the heath senvices utilization.

Fraily it is a condition of hieh vulnerability or of reduced homeostatic efficiency after a stressful event and a consequence of the cumulative decline in the course of Ite in many physiological systems inked to single pathologies or, more frequently, multimorbidities, to their treatment, to lifestyles, to aging.

Disability is the personal condition of those who, following one or more impairments, have a reduced ability to interact with the social environment compared to what is considered the norm, therefore it is less autonomous in carrving out dally activities and often in disadvantaged conditions in participating in social life.

Burden of diseases: Burden of morbidity defines the overall impact of the multimorbidity taking into accoumt the age, the effects of ating, the number and severity of individual diseases, their duration, the frailty and/or disability that ensues.

Complexity The complex condition is caused by the interaction of multiple genes and environmental factors. In geriatriks takes into account of the impact of morbidity burden and extra clinical factors related to health: sex, network and social support, availablity of care, economic, ability to use services, etc

Figure 1. The geriatric canon: conceptual diagram. 


\section{Comorbidity}

Comorbidity is the co-existence of other conditions with an index condition. The term refers to pre-existing or coexisting distinct additional diseases (which appeared at different times or at the same time) with respect to the "index" disease, i.e. the disease that causes a worsening of the state of health in an individual, and/or the acute event or the disease that most affects the prognosis [3-5]. These additional pathologies may be concordant (when they share the same risk factors; i.e. if the index pathology is a myocardial infarction, concordant conditions may be hypertension and dyslipidemia) or cooccurring, but not concordant (i.e. if the index condition is an arrhythmia co-occurring, but not concordant, pathologies may be breast cancer or diverticulosis).

Differentiating the nature of conditions is critical to the conceptualization of comorbidity, because simultaneous occurrence of loosely defined entities may signal a problem with the classification system itself. For example, some would argue that depression and anxiety are not separate entities but part of a spectrum, and, if so, patients with both should not be classified as having comorbidity.

The comorbid diseases may have a variable and independent weight on survival (i.e. COPD, renal cancer) or a variable weight on disability (i.e. polyarthritis, Parkinson's disease) [6] or both. Finally, there are additional conditions that cannot be classified as the previous ones, but that equally can influence the patient's health status (i.e. jatrogenesis, ex-smoking, previous surgery) and that need to be taken into account in the clinical act. The definition of co-morbidity represents an advancement in the understanding of the patient's somatic health: when related to the possible co-presence of other diseases and their severity it allows to define with less uncertainty the therapeutic possibilities, the impact on disability and the prognosis of patient.

Among the available tools some try to quantify the comorbidity severity through modalities that take into account its impact on biological systems (i.e. APACHE of Knaus et al.) [7], other the sum of diseases with different weight on mortality (i.e. Charlson Index) [8], or with different weight on functional status (i.e. Cumulative Illness Rating Scale) [9], or the interaction between number of diseases and their impact on biological systems (i.e. Geriatric Index of Comorbidity) [10].

When we talk about comorbidity, we know what is the index condition and which are the co-morbidities, however it is not only arbitrary, but frequently this is going to change even in a small period of time. For example, the index disease of a patient admitted with a 150-frequency atrial fibrillation is the arrhythmia, the hemodynamic consequences and the associated embolic risk are additional conditions. After cardioversion with an even modest dose of antiarrhythmic drugs, its dominant problem becomes the condition that caused the emergence of atrial fibrillation such as pericarditis, a valvulopathy, poorly controlled hypertension or hyperthyroidism.

\section{Multimorbidity}

The term multimorbidity describes the intercourse or co-presence of two or more chronic diseases or medical conditions in the same individual without attributing to them a causal role or a priority clinical importance. Multimorbidity is the typical condition of a clinically stable elderly patient detected during an outpatient follow-up visit in a general practitioner office $[6,11]$.

In a condition of multimorbidity, chronic diseases can be: i) somatic (i.e. heart failure) or psychic (i.e. depression); ii) concordant (they share the same risk factors: i.e. heart failure and $\mathrm{AF}$, depression and anxiety);

iii) co-occurring and non-concordant (i.e. heart failure and prostate cancer or dementia, depression and psychosis or COPD);

iv) more or less severe (i.e. heart failure NYHA II or IV, COPD Gold II or IV);

Like comorbidities, the conditions that characterize a multimorbidity picture can have:

i) a prevalent impact on survival (i.e. heart failure, COPD) or on disability (i.e. polyarthritis, peripheral neuropathy, dementia);

ii) a short (i.e., lung cancer) or a long evolution (i.e. dysthymia, dementia);

iii) a variable impact on the health services utilization (i.e. COPD, polyarthritis);

Multimorbidity usually lead to a worsening of disability and frailty status, and to a decrease in quality of life.

\section{Frailty}

It is a condition of high vulnerability or of reduced homeostatic efficiency after a stressful event and a consequence of the cumulative decline in the course of life in many physiological systems linked to single pathologies or, more frequently, multimorbidities, to their treatment, to lifestyles, to aging. Frailty is susceptibility to breakage, damage, latent vulnerability, possible loss of adaptive capacity. The etymology of the word fragility comes from "frango", break up, and refers to the notion of something that if subjected to a pressure, to an impact, risks being easily damaged. Frailty is often identified by words that express a similar condition as "vulnerability", or, as more recently occurred, as a condition to be opposed to "robustness" or "resilience" (the capacity to recover quickly from difficulties; the cause of frailty and loss of resilience is probably a progressive loss of redundancy in response patterns and biological adaptive connections) [12].

The concept of frailty is generally linked to that of function, because the risk of loss of the latter is correlated - though not always and not always linearly - with the mechanisms that determine frailty (i.e. the FiND questionnaire presents a very good capacity to correctly identify frail older persons without mobility disability) [13]. There are two concepts of frailty that differ substantially from each other: 1) the "frailty phenotype" conceptualized by Fried and colleagues [14], which includes reduction of muscle strength, fatigue, reduction of walking speed, weight loss (reduced lean mass) and reduced physical activity and 2) the deficit accumulation measured by the "frailty index", conceptualized by Rockwood [15], which includes sensory deficits, disabilities and comorbidities, conditions that are considered related to fragility but which in the same time constitute distinct entities. From these two different concepts of frailty, different scales have been introduced. The two different approaches to "frailty" provide different prognostic information: the "frailty phenotype" gives information about the risk of developing disability in the future, while according to the Rockwood Frailty Index the loss of basal or instrumental functions contributes in itself to the diagnosis of frailty.

\section{Disability}

Disability is the personal condition of those who, following one or more impairments, have a reduced ability to interact with 
the social environment compared to what is considered the norm, therefore it is less autonomous in carrying out daily activities and often in disadvantaged conditions in participating in social life. Disability in the elderly is the main consequence, the final common pathway of chronic diseases and their combination.

Disability is a condition for those who have lasting physical, mental, intellectual or sensory impairments that, in interaction with barriers of different kinds, can hinder their full and effective participation in society. It is the impairment of the person's ability to perform autonomously (albeit with aids) the basic activities of daily life.

Disability in the elderly is the final common pathway of chronic diseases and their combination: Since the prevalence of most chronic conditions increases with age, it also increases the prevalence of disability [16]. Disability has a wide spectrum of manifestations: it can arise both acutely as a consequence of a pathology that has a significant impact on the locomotor system (for example a stroke or a femur fracture) or when the clinical substrate is very frail (for example pneumonia in a malnourished patient with severe comorbidity) or it can progressively appear starting subclinically (characterized by the development of minimal functional limitations, not yet such as to significantly interfere with function): this is the case of non-fatal clinical conditions. The subclinical situation indicates a high risk of subsequent clinically manifest disability.

Functional assessment (of disability) is the main aspect of geriatric evaluation $[17,18]$, it investigates the ability to perform activities, from the simplest to the most complex, and to maintain a social role. In relation to complexity and difficulty the activities of everyday life can be defined as "basic" (BADL: Basic Activities of Daily Living), "instrumental" (IADL: Intermediate Activities of Daily Living) or "advanced" (AADL: Advanced Activities of Daily Living). The basic activities of daily life (BADL) include elementary functions related to the ability to manage one's own person and in particular walking, clothing, food, hygiene, and sphincter control. The intermediate activities of everyday life (IADL) include complex functions such as shopping, managing money, cooking, managing the house, using the telephone. Independence in IADL is important as it often allows us to define whether or not a person can live alone. The advanced activities of everyday life ("Advanced Activities of Daily Living" or AADL) represent more complex activities (and therefore their impairment occurs very early) compared to the BADL and the IADL. The assessment of the ability to perform these activities allows the identification of subjects on the one hand who have a particularly high functional capacity and on the other hand who have modified their behavior by adapting to a slight loss still compatible with the performance of BADL and IADL (examples of AADL are: hobbies, travel, participation in religious or social groups, gardening, sports). These described activities are not essential to maintain independence and considerable individual variations in the ability to perform them must be expected. The used tools (i.e. scales) investigate the ability to perform daily activities by "asking" the subject if he is able to perform them autonomously or if he needs assistance; this characteristic constitutes their main limitation [19-22].

A possible alternative to overcome these limits is provided by objective or "performance" tests, for example, Tinetti balance and gait scale [23]; Reuben PPT-Physical Performance Test [24]; Timed Up and Go Test [25]; SPPB-Short Physical Performance Battery [26]. These consist of asking an individual to perform a specific task by detecting, according to pre-established criteria, some parameters (i.e. how many times the subject is able to perform an action or how long). Performance tests, especially when they contain quantitative assessments (i.e. time taken to complete a task or scores of scales) show a good sensitivity even in the detection of small functional changes, therefore they are the more appropriate tests for the integration of traditional evaluation in measuring the effects not only of pharmacological treatments, but also of surgical procedures on organs or apparatuses not directly related to motor function $[27,28]$.

The functional status of a patient is rarely reported in a clinical record, attributing to him a nursing value rather than a medical one (it is easier to find a description of disability in a nursing record than in a medical one). Yet the functional state is an extraordinary clinical indicator (how can we quantify the severity of a heart failure in a patient unable to walk, at which hemoglobin values a bedridden patient with patient chronic anemia will suffer of dyspnea?). The easiest and quickest way to assess a patient's disability is by asking him/her or relatives a very simple question: can he/she get out of bed alone?

\section{Delirium}

Delirium is an acute, transient, usually reversible, fluctuating disturbance in attention, cognition, and consciousness level. Causes include almost any disorder or drug. Diagnosis is clinical, with laboratory and usually imaging tests to identify the cause. Treatment is correction of the cause and supportive measures.

The diagnosis of delirium represents an umbrella construct adopted to overcome the terminological chaos existing before DSM-III (1980), when dozens of terms were used to indicate generalized brain dysfunction occurring in the context of acute illness or drug intoxication.

After initial assessment, standard diagnostic criteria, such as the Diagnostic and Statistical Manual of Mental Disorders, 5th Edition (DSM-5) [29] or Confusion Assessment Method (CAM), may be used [30].

The following features are required for diagnosis of delirium according to DSM-5 criteria:

i) Disturbance in attention (i.e., reduced ability to direct, focus, sustain, and shift attention) and awareness (reduced orientation to the environment).

ii) The disturbance develops over a short period of time (usually hours to a few days), represents an acute change from baseline attention and awareness, and tends to fluctuate in severity during the course of a day.

iii) An additional disturbance in cognition (i.e.: memory deficit, disorientation, language, visuospatial ability, or perception).

iv) The disturbances in Criteria $\mathrm{A}$ and $\mathrm{C}$ are not better explained by a pre-existing, established or evolving neurocognitive disorder and do not occur in the context of a severely reduced level of arousal such as coma.

v) There is evidence from the history, physical examination or laboratory findings that the disturbance is a direct physiological consequence of another medical condition, substance intoxication or withdrawal (i.e. due to a drug of abuse or to a medication), or exposure to a toxin, or is due to multiple etiologies. CAM uses the following criteria:

i) An altered level of consciousness (i.e. hyperalert, lethargic, stuporous, comatose) or disorganized thinking (i.e., rambling, irrelevant conversation, illogical flow of ideas).

Based on the way the delirium occurs, it can be distinguished in:

ii) Hyperkinetic when it is characterized by anxiety, hyperactivity or aggression (extreme agitation, aggressive, restless);

iii) Hypokinetic when lethargy, hypoactivity, ideomotor slowdown (apathy, sometimes states of unconsciousness) prevail. 
iv) Mixed, when symptoms are characterized by the alternation of hyperkinetic and hypokinetic behaviors [30].

Asking the family if the mental state, or the patient's behavior, has suddenly changed during the illness that motivated the hospitalization is the simplest way to make the screening of the delirium ("delirium", not delirious!).

\section{Dementia}

Dementia is a disorder characterized by the decline in cognition involving one or more cognitive domains (learning and memory, language, executive function, complex attention, perceptualmotor, social cognition). The deficits must represent a decline from previous level of function and be severe enough to interfere with daily function and independence.

Mild cognitive impairment (MCI) is an intermediate clinical state between normal cognition and dementia. While specific subtle changes in cognition can occur in normal aging, MCI can also be a precursor to dementia. At the same time, MCI may also represent a reversible condition in the setting of depression, as a complication of certain medications, or during the recovery from an acute illness.

Alzheimer's disease (AD) is the most common cause of dementia, $10-20 \%$ is vascular, while other neurodegenerative diseases, such as Lewy's disease, frontotemporal dementia are the cause of dementia in $10-30 \% \%$ of cases. A limited number of cases $(1-2 \%)$ are secondary to potentially reversible pathologies.

The proposed definition of dementia applies to all diseases associated to cognitive decline and is particularly useful both on the classification and the clinical level.

Dementia was named major neurocognitive disorder (NCD) in the DSM-5 [29]. However, the term dementia may still be used as an acceptable alternative. The two terms are essentially different labels for the same condition; major NCD is equivalent to dementia.

DSM-5 criteria for major neurocognitive disorder (dementia) include:

i) Evidence of significant cognitive decline from a previous level of performance in one or more cognitive domains (complex attention, executive function, learning and memory, language, perceptual-motor, or social cognition) based on:

1) Concern of the individual, a knowledgeable informant, or the clinician that there has been a significant decline in cognitive function; and

2) A substantial impairment in cognitive performance, preferably documented by standardized neuropsychological testing or, in its absence, another quantified clinical assessment.

ii) The cognitive deficits interfere with independence in everyday activities (that is, at a minimum, requiring assistance with complex instrumental activities of daily living such as paying bills or managing medications).

iii) The cognitive deficits do not occur exclusively in the context of a delirium.

iv) The cognitive deficits are not better explained by another mental disorder.

Specify:

- Without behavioral disturbance: if the cognitive disturbance is not accompanied by any clinically significant behavioral disturbance;

- With behavioral disturbance (specify disturbance): if the cognitive disturbance is accompanied by a clinically sig- nificant behavioral disturbance (for example, psychotic symptoms, mood disturbance, agitation, apathy, or other behavioral symptoms)

The differentiation of dementia from mild clinical deterioration (Mild Cognitive Impairment: MCI) is based on determining whether or not there is significant interference with the ability to function at work or in the usual daily activities. This is of course a clinical judgment, carried out by an expert doctor on the basis of the patient's individual circumstances and by the description of daily events obtained by the patient and by a subject who knows him.

Asking a patient (or his family members) whether he/she is autonomous in taking medication (usually very numerous) or if he/she needs help is an effective way and does not require additional time to the visit in order to detect a probable cognitive impairment.

\section{Depression}

The essential characteristic of depression at all ages is dysphoric mood or loss of pleasure or interest in normal activities. This disorder is prominent and persistent and is accompanied by a number of typical symptoms and signs. For a thorough diagnosis of the diagnostic category of depressive disorder, which in some cases must be done with the help of a psychiatrist, it is necessary to evaluate the severity and duration of symptoms, as outlined below [29]:

- Feelings of sadness, tearfulness, emptiness or hopelessness

- Angry outbursts, irritability or frustration, even over small matters

- Loss of interest or pleasure in most or all normal activities, such as sex, hobbies or sports

Sleep disturbances, including insomnia or sleeping too much

- Tiredness and lack of energy, so even small tasks take extra effort

- Reduced appetite and weight loss or increased cravings for food and weight gain

- Anxiety, agitation or restlessness

- Slowed thinking, speaking or body movements

- Feelings of worthlessness or guilt, fixating on past failures or self-blame

- Trouble thinking, concentrating, making decisions and remembering things

- Frequent or recurrent thoughts of death, suicidal thoughts, suicide attempts or suicide

- Unexplained physical problems, such as back pain or headaches

Depressive symptomatology may be the psychic epiphenomenon of a general condition of inadequacy, of psychological, physical, relational, socio-environmental incompetence. Chronic diseases, chronic pain syndromes, recent changes in life and the presence of disadvantageous conditions, the presence of poor health, and unexplained physical symptoms are associated with depression. The probability of a depressive disorder increases by about 1.5 to 3.5 times if any of these factors is present. This explains the wide variability in symptoms, which in turn is secondary to the variability of interconnecting risk factors and the consequent classification difficulties have led most clinicians to operationally consider depression as a continuum of illness, which varies in severity and duration, rather than a condition to be classified by strict diagnostic criteria [31].

The simple question "Have you been sad or depressed most of the time in the last year?" is a good starting point for identifying a patient with a depressive disorder. 


\section{Burden of morbidity}

Burden of morbidity defines the overall impact of the multimorbidity taking into account the age, the effects of aging, the number and severity of individual diseases, their duration, the frailty and / or disability that ensues [5].

\section{Complex condition}

The complex condition is caused by the interaction of multiple genes and environmental factors. In geriatrics, this concept considers the impact of morbidity burden and extra-clinical factors related to health: sex, network and social support, availability of care, economic, ability to use services, etc. [5].

Network and social support affect the patient's health status and its vital trajectory. Social support is assistance provided through a social network and its positive perception. Social support is a buffer to stress as well as an element of moderation of physical and mental well-being. Patients who do not have a meaningful social network may not have the necessary information or feedback, while people who have adequate social support may be able to cope with the implicit changes in their aging and related crises (medical and social). Social support can be divided into emotional support, information and advice, tangible help, social stimulation. Daily support is that provided when needed [28]. While age related systems changes, clusters of pathological conditions (comorbidity and multimorbidity), syndromes, frailty, disability, burden of diseases, network and social support have been described individually, in reality they coexist, develop in parallel and interfere with each other through feed-back and feed-forward loops: the phenotype of aging (the characteristics of the patient) is the result of their interactions.

\section{Conclusions}

Under conditions of complexity due to uncertainty, it is easy to say that having a series of defined elements can lead to better decisions that will determine the quality of care and at the same time that there are elements related to the professional that is treating each case.

According to evidence-based medicine the clinician identifies the index condition, asks the appropriate question, searches for and trace the right evidence and appropriately applies the evidence that has been found (three elements make the characteristics of a medical doctor: clinical competence, the ability to communicate with other experts and to work in a team).

Today the complexity and the facing of new diagnostic tools and therapies require an improved set of skills necessary to face all the different challenges, and therefore it is necessary to talk with those who have more in-depth skills in other specialties and then work as a team. Every industry requires a glossary that is more precise and intelligible, which indicates to each component of the team specific thoughts, information and a speed at which they can be transmitted. This need to be applied in the medical areas where health is the result of biomedical, functional, social and relational factors such as those dealing with aging and related diseases. If the concepts are clear, information can be communicated between the members of the care team and therefore the diagnostic communion can be fulfilled, furthermore the definition of the goals and the tools to obtain them can be more easily identified and shared.

\section{References}

1. Bernabei R, Gray L, et al. International Gerontology. Hazzard's principles of geriatric medicine and gerontology, 6th Edition. New York: McGraw-Hill; 2009.

2. Ferrucci L, Studenski S. Clinical problems of aging. In: Kasper DL, Fauci AS, Hauser SL, Longo DL, Jameson JL, Loscalzo J, editors. Harrison's principles of internal medicine, 19th ed. New York: McGraw-Hill; 2015.

3. Feinstein AR. The pretherapeutic classification of comorbidity in chronic disease. J Chronic Dis 1970; 23:455-468.

4. van den Akker M, Buntinx F, Knottnerus JA. Comorbidity or multimorbidity: What's in a name? A review of literature. Eur J Gen Pract 1996; 2:65-70.

5. Valderas JM, Starfield B, Sibbald B, et al. Defining comorbidity: implications for understanding health and health services. Ann Fam Med 2009;7:357-63.

6. Academy of Medical Sciences. Multimorbidity: a priority for global health research. 2018. Available from: https://acmedsci.ac.uk/policy/policy-projects/multimorbidity

7. Knaus WA, Draper EA, Wagner DP, et al. APACHE II: a severity of disease classification system. Crit Care Med 1985; 13:818-29.

8. Charlson ME, Pompei P, Ales KL et al. A new method of classifying prognostic comorbidity in longitudinal studies: Development and validation. J Chronic Dis 1987; 0:373-83.

9. Parmelee PA, Thuras PD, Katz IR, Lawton MP. Validation of the Cumulative Illness Rating Scale in a geriatric residential population. J Am Geriatr Soc 1995;43:130-7.

10. Rozzini R, Frisoni GB, Ferrucci L, et al. Geriatric index of comorbidity: Validation and comparison with other measures of comorbidity. Age Ageing 2002;31:277-85.

11. Marengoni A, Rizzuto D, Wang HX, et al. Patterns of chronic multimorbidity in the elderly population. J Am Geriatr Soc 2009;57:225-30.

12. Ferrucci L, Giallauria F, Schlessinger D. Mapping the road to resilience: novel math for the study of frailty. Mech Ageing Dev 2008;129:677-9.

13. Cesari M, Demougeot L, Boccalon H, et al. A self-reported screening tool for detecting community-dwelling older persons with frailty syndrome in the absence of mobility disability: the FiND questionnaire. PLoS One 2014;9:e101745.

14. Fried LP, Tangen CM, Walston J, et al. Frailty in older adults: evidence for a phenotype. J Gerontol Med Sci 2001;56:M146-56.

15. Rockwood K, Stolee P, McDowell I. Factors associated with institutionalization of older people in Canada: testing a multifactorial definition of frailty. J Am Geriatr Soc 1996; 44:578-82.

16. Freeman D, Garety PA, Kuipers E, et al. A cognitive model of persecutory delusions. Br J Clin Psychol 2002;41:331-47.

17. Kane RA, Kane RL. Assessing the elderly: A practical guide to measurement. Lexington, 1981.

18. Kane RL, Kane RA. Assessing older persons: Measures, meaning, and practical application. New York: Oxford University Press; 2000.

19. Mahoney FI, Barthel W. Functional Evaluation: the Barthel Index. Mar St Med J 1965;14:61-5.

20. Katz S, Downs TD, Cash HR, et al. Progress in the development of the index of ADL. Gerontologist 1970;10:20-30. 
21. Lawton MP, Brody EM. Assessment of older people: selfmaintaining and instrumental activities of daily living. Gerontologist 1969;9:179-86.

22. Reuben DB, Laliberte L, Hiris J, Mor V. A hierarchical exercise scale to measure function at the Advanced Activities of Daily Living (AADL) level. J Am Geriatr Soc 1990;38:855-61.

23. Tinetti ME. Performance-oriented assessment of mobility problems in elderly patients. J Am Geriatr Soc 1986;34:119-26.

24. Podsiadlo D, Richardson S. The timed "Up \& Go": a test of basic functional mobility for frail elderly persons. J Am Geriatr Soc 1991;39:142-8.

25. Reuben DB, Sin AL. An objective measure of physical function of elderly outpatients. The Physical Performance Test. J Am Geriatr Soc 1990;38:1105-12.

26. Guralnik JM, Simonsick EM, Ferrucci L, et al. A short physical performance battery assessing lower extremity function: asso- ciation with self-reported disability and prediction of mortality and nursing home admission. J Gerontol 1994;49:M85-94.

27. Guralnik JM, Branch LG, Cummings SR, Crib JD. Physical performance measures in aging research. J Gerontol 1989;44: 141-46.

28. Rozzini R, Ferrucci L, Barbisoni P, et al. The effect of chronic diseases on physical function. Comparison between activities of daily living scales and the Physical Performance Test. Age Ageing 1997;26:281-287.

29. American Psychiatric Association. Diagnostic and statistical manual of mental disorders. 5th ed. Arlington: American Psychiatric Publishing; 2013.

30. Inouye SK, Westendorp RG, Saczynski JS. Delirium in elderly people. Lancet 2014;383:911-22.

31. Rozzini R, Bianchetti A, Trabucchi M. Medicina della fragilità: manuale di lavoro. Milan: Vita e Pensiero; 2014. 